\title{
Theta graphs, graph decompositions and related graph labelling techniques
}

\author{
ANDREW BLINCO
}

In recent years much work has been done on finding edge-disjoint decompositions of a complete graph on $v$ vertices, $K_{v}$, into copies of certain graphs. In particular, the problem of finding decompositions of complete graphs into cycles of length $n, C_{n}$, has received much attention, and this problem was solved for many cases by Rosa [5], Lindner and Rodger [3]. Recently, the necessary conditions for the existence of a decomposition of the complete graph into cycles of a fixed length have been shown to be sufficient by Alspach and Gavlas [1] and Sajna [6]. For any graph $G$ the set of values of $v$ for which there exists a $G$-decomposition of $K_{v}$ (also called a $G$-design of order $v$ ) is called the spectrum for $G$-designs. In this thesis we consider the spectrum problem for certain theta graphs. A theta graph is a simple graph consisting of two vertices joined by a number of internally disjoint paths, and will be denoted by $\Theta\left(l_{1}, l_{2}, \ldots, l_{t}\right)$, where $l_{1}, l_{2}, \ldots, l_{t}$ are the lengths of the paths. This notation for theta graphs was introduced by Loerinc [4]. As an example, $K_{4}-e$, a complete graph on four vertices missing one edge, is a theta graph consisting of four vertices, with two vertices joined by one path of length one and two paths of length two, denoted by $\Theta(1,2,2)$. Also, note the graphs $K_{m+2} \backslash K_{m}$ constitute a family of theta graphs. Many properties of the graph $K_{4}-e$ (and also the graphs $K_{m+2} \backslash K_{m}$ ) have been investigated. The spectrum problem for theta graphs consisting of three paths will be the focus of much of this thesis. Theta graphs of the form $\Theta\left(l_{1}, l_{2}, l_{3}\right)$ may be thought of as an edge-amalgamation of two cycles of length $l_{i}+l_{j}$ and $l_{i}+l_{k}$, with $l_{i}$ edges in common, where $(i, j, k)$ is some permutation of $(1,2,3)$. Continuing with the previous example, $\Theta(1,2,2)$ may be thought of as an amalgamation of two cycles of length three, with one edge in common.

Unlike cycle designs, the obvious necessary conditions for the existence of a theta graph design are not always sufficient; and this lack of sufficiency in certain cases is investigated in Chapter 2.

In Chapters 3, 4 and 5 we complete the spectrum problem for theta graphs with fewer than ten edges. Many of the necessary examples (which appear in Appendix A)

Received 15th September, 2003

Paper submitted to The University of Queensland, February 2003. Degree approved, July 2003. Supervisor: Dr Elizabeth Billington.

Copyright Clearance Centre, Inc. Serial-fee code: 0004-9727/04 \$A2.00+0.00. 
used these in chapters were found using autogen, a graph decomposition software package written by Adams.

A decomposition of $K_{v} \backslash L$, the complete graph of order $v$ with a graph $L$ (called the leave) removed, into edge disjoint copies of a graph $G$ is called a maximum packing of $K_{v}$ with $G$ if $L$ contains as few edges as possible. A decomposition of $K_{v} \cup X$ with $V(X) \subseteq V\left(K_{v}\right)$, the complete graph of order $v$ union a graph $X$ (called the excess), into edge disjoint copies of a graph $G$ is called a minimum covering of $K_{v}$ with $G$ if $X$ contains as few edges as possible. The maximum packing and minimum covering problems have been studied for many graphs. In Chapter 6 we construct maximum packings and minimum coverings of $K_{v}$ with copies of $\Theta(1,3,3)$ for all $v \not \equiv 0$ or $1(\bmod 7)$, where $L$ and $X$ are compact (that is, are simple graphs on the least number of vertices). The necessary examples required for the constructions given in this chapter can be found in Appendix B. (When $v \equiv 0$ or $1(\bmod 7)$, the leave or excess is empty, and a decomposition of $K_{v}$ in this case into $\Theta(1,3,3)$ is covered by the results of Chapter 3.)

In Chapters 7, 8 and 9 we present some general constructions for theta graph decompositions. In Chapter 7 , methods for decomposing the complete graph $K_{v}$ into theta graphs of the form $\Theta(1, n, n)$ are given when $v \equiv 0(\bmod 2 n+1)$ if $n$ is odd, and when $v \equiv 1(\bmod 2 n+1)$, if $n \equiv 3(\bmod 4)$; in the case $n \equiv 1(\bmod 4)$ further partial results are obtained. In Chapter 8 methods for decomposing the complete graph $K_{v}$ into theta graphs of the form $\Theta(1, n, n)$ are given when $v \equiv 1(\bmod 2 n+1)$ and $n \equiv 2(\bmod 4)$. In Chapter 9 methods for decomposing the complete graph $K_{v}$ into theta graphs of the form $\Theta(1,2 m, 2 n-2 m)$ are given when $v \equiv 1,2 n+1,2 n+2(\bmod 4 n+2)$ and $n$ is odd.

For any graph $G$, an injective function $h: V(G) \rightarrow \mathbb{N}$ is called a labelling (or a valuation) of $G$. In 1966, Rosa introduced a hierarchy of labellings, including $\rho-, \beta$ and $\alpha$-labellings. In Chapters 10 and 11 some new types of labellings are added to this hierarchy. Let $\rho^{+}$denote an ordered $\rho$-labelling. As with the case of an $\alpha$-labelling, a $\rho^{+}$-labelling of a graph $G$ with $n$ edges yields a cyclic $G$-decomposition of $K_{2 n x+1}$ for all positive integers $x$. It was shown by El-Zanati, Vanden Eynden and Punnim [2] that the disjoint union of a collection of graphs with $\alpha$-labellings has a $\rho^{+}$-labelling. In Chapter 10 we show that the disjoint union of a collection of graphs with $\alpha$-labellings together with a graph with a slightly restricted $\rho^{+}$-labelling (called a $\rho^{++}$-labelling) also has a $\rho^{++}$. labelling. This result has an application in the cyclic decomposition of certain complete graphs into the disjoint union of even-length cycles.

An almost-bipartite graph is a non-bipartite graph with the property that the removal of a particular single edge renders the graph bipartite. Examples of such graphs include odd cycles and the graphs $K_{m+2} \backslash K_{m}$. The technique of labelling the vertices of bipartite graphs to yield decompositions of certain complete graphs has received much attention in the literature. In Chapter 11 we introduce the concept of a $\gamma$-labelling of an almostbipartite graph and show that if an almost-bipartite graph $G$ with $n$ edges has a $\gamma$ - 
labelling then there is a cyclic $G$-decomposition of $K_{2 n x+1}$ for all positive integers $x$. We show that odd cycles and certain other almost-bipartite graphs have $\gamma$-labellings.

In Chapter 12, we combine the results concerning theta graph designs with the results concerning the labelling of the vertices of graphs by giving $\alpha$-labellings of bipartite theta graphs and $\gamma$-labellings of some almost-bipartite theta graphs.

In Chapter 13, a small result concerning Eulerian circuits is presented.

Finally, in Chapter 14 we summarize the results of this thesis.

\section{REFERENCES}

[1] B. Alspach and H. Gavlas, 'Cycle decompositions of $K_{n}$ and $K_{n}-I$ ', J. Combin. Theory Ser. B 81 (2001), 77-99.

[2] S.I. El-Zanati, C. Vanden Eynden and N. Punnim, 'On the cyclic decomposition of complete graphs into bipartite graphs', Australas. J. Combin. 24 (2001), 209-219.

[3] C.C. Lindner and C.A. Rodger, 'Decomposition into Cycles II: Cycle Systems', in Contemporary Design Theory: A Collection of Surveys, (J.H. Dinitz and D.R. Stinson, Editors) (J. Wiley and Son, New York, 1992), pp. 325-369.

[4] B. Loerinc, 'Chromatic uniqueness of the generalised $\theta$-graph', Discrete Math. 23 (1978), 313-316.

[5] A. Rosa, 'On certain valuations of the vertices of a graph', in Théorie des graphes, journées internationales d'études, Rome 1966 (Dunod, Paris, 1967), pp. 349-355.

[6] M. Šajna, 'Cycle decompositions III. Complete graphs and fixed length cycles', J. Combin. Des. 10 (2002), 27-78.

Department of Mathematics

The University of Queensland

Queensland 4072 Australia

e-mail: ablinco@stvcas.cas.ilstu.edu 